\title{
The Doppler Factor and Quantum Electrodynamics Basics in Laser-Driven Light Sailing
}

\author{
Gabriel James Tambunga \\ 916 S. Delhi Street, Philadelphia, PA 19147, U.S.A. \\ E-mail address: mexicanengineer911@gmail.com
}

\begin{abstract}
Basic concepts in Quantum Electrodynamics (QED) and the doppler factor were applied to laser-driven light sailing, where this combination was not considered before. QED indicates that a reflection is an absorption and an emission of discreet energy, and the doppler factor indicates a change in velocity of a reflector would result in the reflector observing a change in wavelength from a source. Applying these concepts results in the reflectors available discrete energy states not being filled, after the reflector changes its velocity. Changes in the apparatus of the laser-driven light sail and changes in the current understanding of the transfer of momentum from light to matter for effective laser-driven light sailing are suggested.
\end{abstract}

Keywords: Quantum Electrodynamics; Doppler Factor; Laser-Driven Light Sailing

\section{INTRODUCTION}

In 1966 it was theorized that space traveling was possible by the use of lasers [1]. This idea was re-enforced by further calculations [2,3] and remains a possibility [4]. This idea is based on the source, the laser, having a constant wavelength while the reflector results in a change in momentum after a reflection; the change in momentum of a reflector can result in a doppler shift, where the reflector, from the reflectors perspective, would view a larger wavelength, or decrease in frequency, of the lasers photons. The doppler shift was previously considered [5,6]. However, Quantum Electrodynamics indicates a reflection is two events, an absorption and emission of a photon, where the energy that is absorbed and emitted is discreet [7,8]; and an absorption of a photon is based on available energy states of an atom [9-11], or for a reflector for this paper. This implies the reflector may not reflect the lasers emissions if the change in momentum of the reflector results in wavelengths that are too low in energy, from the reflectors perspective, for the available energy states, of the reflector, which are used in reflection. Laser cooling considered this by using lasers of increasing frequencies to slow down particles. That is, when a particle is approaching a source, the particles view the lasers emissions as higher in energy, so the laser must have a lower energy emission so the particles 
discreet available energy states can be filled for a reflection to occur; and after the reflection, to slow the slower particle further, the lasers emissions would need to be slightly increased but still lower in energy, as compared to the particle at the same moving reference frame as the source, for another reflection to occur $[12,13]$. In this paper the doppler effect of a reflection will be discussed with the consideration that the reflectors available energy states will be unchanging from the perspective of the reflector. The results of this paper will suggest changes in the apparatus for laser travel in space.

\section{ABSOPTION AND EMISSION}

The source, or laser, for interstellar laser travel would need to change so the reflector can view the frequency of the photons, from the source, to be consistent if the source is to continually provide momentum to the reflector. If the reflector requires frequency $f_{r}$, then the source frequency would be

$$
\sqrt{\frac{1+\beta}{1-\beta}} f_{r}
$$

which is acquired from the doppler factor

$$
\sqrt{\frac{1+\beta}{1-\beta}}=\frac{f_{s}}{f_{o}},
$$

where $\beta=\mathrm{v} / \mathrm{c}, f_{\mathrm{s}}$ is the frequency from the source and $f_{\mathrm{o}}$ is the observed frequency.

Equation (a) represents the frequency relationships for the absorption part of the reflection. For absorption, the energy of the photon is temporarily stored in the atoms/molecules, leaving no excess energy for the reflector to be in motion. For an emission there is a drop in energy state, of the atoms/molecules, which is equal to the energy of the emission, where again there is no excess energy for the reflector to be in motion.

\section{CONSERVATION OF ENERGY}

Considering only the energy, there would be no motion resulting from the absorption or emission from the reflectors perspective.

However, the energy from the source, from the sources perspective, is not equal to the energy from the reflector. That is, the frequency from the reflector would be

$$
\sqrt{\frac{1-\beta}{1+\beta}} f_{r} .
$$

Though energy is conserved from the reflectors perspective, the source would view a loss of

$$
\frac{2 \beta}{\sqrt{1-\beta^{2}}} E_{r},
$$


from the energy emitted and received, where $\mathrm{E}_{\mathrm{r}}=h f_{\mathrm{r}}$.

To determine where the loss of energy went to, the momentum of the photons will be considered. Using the relations $\mathrm{p}=h / \lambda$ and $\mathrm{c}=\lambda f$, the momentum from the source is

$$
\sqrt{\frac{1+\beta}{1-\beta}} \frac{h}{\lambda_{r}},
$$

the momentum the reflector sees is $h / \lambda_{\mathrm{r}}$, and from the sources perspective, the reflector emits

$$
\sqrt{\frac{1-\beta}{1+\beta}} \frac{h}{\lambda_{r}} .
$$

Considering parallel vectors, as the vectors are in opposite directions the resultant vector would be

$$
\frac{2}{\sqrt{1-\beta^{2}}} \frac{h}{\lambda_{r}},
$$

pointing in the direction of the source photon. This implies the energy can be transferred to motion from the sources perspective. However, for the conservation of energy to be conserved, kinetic energy resulting from a reflection occurs as a result of the doppler shift from the sources perspective and not a direct exchange of energy between photon and reflector.

\section{CONSERVATION OF MOMENTUM}

For the conservation of momentum to be conserved there are two viewpoints that will be considered.

\section{1. Viewpoint One}

The first viewpoint is the current belief that there is a transfer of momentum from the photons to the reflector upon absorption [9-11], where the reflectors momentum would be $h / \lambda_{\mathrm{r}}$ in its higher energy state, from the reflectors perspective. From this same perspective, recoil of the reflector, after emission, would change the momentum to $2 h / \lambda_{\mathrm{r}}$, as the momentum acquired from the recoil is equal to the momentum of the emitted photon. Further, as the emission and recoil would happen at the same time, the momentum of the returning photon, from the sources perspective, would be

$$
\sqrt{\frac{1-\beta^{\prime}}{1+\beta^{\prime}}} \frac{h}{\lambda_{r}}
$$

where:

$$
\beta^{\prime}=\frac{v}{c}+\frac{h}{m \lambda_{r}}
$$


and $\mathrm{m}$ is the mass of the reflector.

The momentum of the reflector, from the sources perspective, would then be

$$
\sqrt{\frac{1+\beta}{1-\beta}} \frac{h}{\lambda_{r}}+\sqrt{\frac{1-\beta^{\prime}}{1+\beta^{\prime}}} \frac{h}{\lambda_{r}}
$$

Comparing the increase in momentum from the reflectors perspective to the sources perspective yields

$$
\frac{2 h}{\lambda_{r}}=\sqrt{\frac{1+\beta}{1-\beta}} \frac{h}{\lambda_{r}}+\sqrt{\frac{1-\beta^{\prime}}{1+\beta^{\prime}}} \frac{h}{\lambda_{r}}
$$

That is, despite velocity of the reflector or wavelength of the photon from the source, the momentum would not go beyond $2 h / \lambda_{\mathrm{r}}$. Simple spreadsheet calculations indicate

$$
\frac{2 h}{\lambda_{r}} \neq \sqrt{\frac{1+\beta}{1-\beta}} \frac{h}{\lambda_{r}}+\sqrt{\frac{1-\beta^{\prime}}{1+\beta^{\prime}}} \frac{h}{\lambda_{r}}
$$

for all values of velocity, v, or source wavelength. Similarly, comparing the energy gain of the reflector, $h^{2} / \mathrm{m} \lambda_{\mathrm{r}}^{2}$, after recoil from the reflectors perspective, to the photon energy before and after reflection, from the sources perspective, yields

$$
\frac{h^{2}}{m \lambda_{r}^{2}} \neq \sqrt{\frac{1+\beta}{1-\beta}} E_{r}-\sqrt{\frac{1-\beta^{\prime}}{1+\beta^{\prime}}} E_{r}
$$

for all values of velocity, v, or source frequency after simple spreadsheet calculations. From this viewpoint, conservation of momentum and energy are not always conserved.

\section{2. Viewpoint Two}

The second viewpoint has not been considered before, to the authors knowledge. That is, momentum is absorbed into the energy state of the atoms/molecules, and does not transfer to the atom/molecules distinctly from the energy; the emitted vector from the reflector changes the speed and direction of the universe, from the reflectors perspective, if the vector leaving the reflector is not identical to the absorbed vector, where the absorbed vector originates from a moving source; and the energy lost from the sources perspective is equal to the energy gained in the reflectors perspective, and vice versa for a mirror slowing down due to laser emissions. From this viewpoint, the momentum and kinetic energy of the reflector, after a reflection, are results of a doppler shift, from the sources perspective; thereby, conserving energy and momentum.

\section{CONCLUSION}

Equations (a) to (f) can be applied to a reflector moving away from a source, to increase its energy and momentum, and a reflector moving toward a source, to decrease its energy and momentum; where the sign of the velocity is positive if the reflector is moving in the direction of the sources emissions and negative if moving towards. The equations also imply that a 
monochromatic source may not be adequate for a reflector intended to increase or decrease in velocity, as the reflector would see different wavelengths than the source emits after a change in the reflectors velocity. The source photons would need to change in wavelength and/or the reflector must be capable of reflecting multiple wavelengths, possibly beyond the visible spectrum.

These equations and the second viewpoint are not consistent with current beliefs $[6,10]$, but can be easily proven or disproven, which is beyond this theoretical introduction presented in this paper. However, if a reflector can be designed to maintain an adiabatic excited energy state for a measurable time, then an increase in the velocity of the reflector would be at about half this time according to the first viewpoint, or at about this time according to the second viewpoint.

\section{References}

[1] G. Marx, Nature 211 (1966) 22-23.

[2] J. L. Redding, Nature 213 (1967) 588-589.

[3] J. F. L. Simmons, C. R. Mcinnes, Am. J. Physics 61 (1993) 205-207.

[4] Y. K. Bae, Physics Procedia 38 (2012) 253-279.

[5] A. Macleod, http://arxiv.org/abs/physics/0407077 (2004) 22 pages.

[6] C. R. Mcinnes, Solar Sailing: Technology, Dynamics and Mission Applications, Springer-Verlag, London, (2004).

[7] M. Fox, Quantum Optics, An Introduction, Oxford University Press, New York, (2006).

[8] V. B. Berestetskii, L. P. Pitaevskii, Quantum Electrodynamics, Elsevier, Burlington, MA, (1982), ed. 2: vol. 4.

[9] G. S. Summy, B. Lohmann, W. R. MacGillivray, M. C. Standage, Z. Phys. D. 30 (1994) 155-159.

[10] G. S. Summy, W. R. MacGillivray, M. C. Standage, J. Phys. B: At. Mol. Opt. Phys. 29 (1996) 2607-2628.

[11] G. S. Summy, B. T. H. Varcoe, W. R. MacGillivray, M. C. Standage, J. Phys. B: At. Mol. Opt. Phys. 30 (1997) L541-L549.

[12] T. W. Hansch, A. L. Schawlow, Optics Communication 13 (1975) 68-69.

[13] W. D. Phillips, H. Metcalf, Physics Review Letters 48 (1982) 596-599. 